\title{
Capacity Analysis and Power Allocation over Non-identical MISO Rayleigh Fading Channels
}

\author{
Le $\mathrm{Cao}^{1}$, Meixia $\mathrm{Tao}^{1,2}$ and Pooi Yuen $\mathrm{Kam}^{1}$ \\ ${ }^{1}$ Dept. of Electrical \& Computer Engineering, National University of Singapore, Singapore 117576 \\ ${ }^{2}$ Dept. of Electronic Engineering, Shanghai Jiao Tong University, Shanghai, China 200240 \\ Emails: caole@nus.edu.sg; mxtao@ieee.org; elekampy@nus.edu.sg
}

\begin{abstract}
We analyze the capacity of a multiple-input singleoutput system over Rayleigh fading channels. The channels are assumed to be independent and non-identically distributed. Simple, explicit and closed-form expressions of ergodic mutual information and outage probability are obtained. Moreover, two suboptimal but efficient analytical power allocation schemes for mutual information maximization and outage minimization are derived, respectively. In specific, for mutual information maximization, more power is assigned to those channels with higher channel variances, while for outage minimization the power allocation scheme follows the water-filling principle.
\end{abstract}

\section{INTRODUCTION}

Multiple-input multiple-output (MIMO) technology has attracted attention in wireless communications, since it offers significant increases in data throughput and link reliability without additional bandwidth or transmit power. Tremendous research work has been done on co-located MIMO systems, where identical channel distribution is often assumed. Recently, MIMO systems over non-identical fading channels have also attracted great attention because of their application in cooperative communications and distributed antenna systems. In decode-and-forward (DF) cooperative communications systems [1], a transmission from a source to a destination is facilitated with the help of a set of relays. In the second stage when those relays have known the transmitted signal, the subsequent transmission can be regarded as a non-identical multi-input single-output (MISO) system. In distributed antenna systems [2], multiple antennas work with the help of coaxial cable to simulcast signals. A non-identical MISO system actually is formed, which enhances signal quality, increases system capacity and improves coverage. Unlike conventional MIMO systems, non-identical MIMO systems experience independent but not necessarily identically distributed (i.n.d) fading. Effects of i.n.d fading in MIMO systems have been studied on several aspects. The bit-error performances over i.n.d Rayleigh/Ricean and Nakagami fading channels using space-time block codes (STBC) are studied in [3] and [4], respectively. In [5], the biterror performance over semi-identical Rayleigh fading channels using differential STBC with noncoherent receivers is analyzed. On the other hand, authors in [6] show that, for ergodic capacity maximization, the eigenvalues of the covariance matrix of input signals are monotonically distributed with respect to the ordered singular values of non-identical MIMO channel matrix. Furthermore, the total power is only shared by those eigen-channels with large enough singular values.
The outage probability of mutual information over distributed MISO systems with i.n.d Rayleigh fading is investigated in [7]. Therein, a heuristic power control scheme named equal power allocation with channel selection is proposed and is near optimal in minimizing the outage probability.

In this work, we evaluate both ergodic mutual information and outage probability over MISO i.n.d Rayleigh fading channels. Simple, explicit, and closed-form expressions at any given power allocation are derived and can be easily extended to multiple-receiver case. While equal power control is capacity-achieving for independent and identically distributed (i.i.d) channels [8], it is not so for i.n.d channels. Our contribution lies in the derivations of two suboptimal power allocation schemes, with only channel statistical information available at the transmitter, for ergodic mutual information maximization and outage minimization, respectively. In particular, the derivation of the power allocation for outage minimization is based on Chernoff bound and different from [7].

\section{SySTEM MODEL}

Consider a narrowband system with $N_{t}>1$ transmit antennas and a single receive antenna. The channel is Rayleigh fading with additive white Gaussian noise of power spectral density $N_{0}$. The channel coefficient from the $i^{t h}$ transmit antenna is denoted by $h_{i}$ and $\left\{h_{i}\right\}_{i=1}^{N_{t}}$ is a set of independent zero-mean complex Gaussian variables, each with variance $\sigma_{i}^{2}$. Without loss of generality, we order the channel gains in a non-increasing order, i.e. $\sigma_{1}^{2} \geq \sigma_{2}^{2} \geq \cdots \geq \sigma_{N_{t}}^{2}$. The transmit signals from different antennas are taken to be Gaussian and independent of each other. Let $p_{i}$ denotes the transmit power on the $i^{\text {th }}$ antenna which is subject to a normalized total power constraint $\sum_{i=1}^{N_{t}} p_{i}=1$. For a given power vector $\mathbf{p}=\left(p_{1}, \ldots, p_{N_{t}}\right)$ and channel realization $\mathbf{h}=\left(h_{1}, \ldots, h_{N_{t}}\right)$, the instantaneous mutual information can be written as

$$
I(\mathbf{p}, \mathbf{h})=\log _{2}\left(1+\gamma_{e}\right),
$$

where $\gamma_{e}$ is called the instantaneous effective signal-to-noise ratio and given by

$$
\gamma_{e}=\frac{1}{N_{0}} \sum_{i=1}^{N_{t}} p_{i}\left|h_{i}\right|^{2} .
$$




\section{ERGODIC MUTUAL INFORMATION AND POWER ALLOCATION}

\section{A. Ergodic mutual information analysis}

The ergodic mutual information of the considered channel model is given by

$$
\bar{I}(\mathbf{p})=E\left[\log _{2}\left(1+\gamma_{e}\right)\right],
$$

where the expectation $E[\cdot]$ is taken over all realizations of h. From (2) it is clear that $\gamma_{e}$ is a weighted sum of $N_{t}$ independent and normalized chi-square random variables with weights $p_{i} \sigma_{i}^{2} / N_{0}$. Its probability distribution function (PDF) can thus be expressed as $[9,(1)]$

$$
f\left(\gamma_{e}\right)=\left.\sum_{k} \frac{-N_{0} e^{-x / \lambda_{k}}}{\left(-\lambda_{k}\right)^{m_{k}}} \frac{g_{k}^{\left(m_{k}-1\right)}(0, x)}{\left(m_{k}-1\right) !}\right|_{x=N_{0} \gamma_{e}}
$$

where $\left\{\lambda_{k}\right\}_{k=0}^{N^{\prime}-1}$ represent the $N^{\prime}\left(\leq N_{t}\right)$ distinct values, each with multiplicity $m_{k}$, as a result of reordering and grouping the $N_{t}$ values of $p_{i} \sigma_{i}^{2}$ for $i=1, \cdots, N_{t} ; \alpha_{k l}$ is defined as $\alpha_{k l}=$ $1-\lambda_{l} / \lambda_{k}$; and $g_{k}^{\left(m_{k}-1\right)}(s, x)$ denotes the $\left(m_{k}-1\right)^{t h}$ derivative of $g_{k}(s, x)$ given in (5) with respect to $s$.

$$
g_{k}(s, x)=e^{-s x} \prod_{l \neq k} \frac{1}{\left(\alpha_{k l}-\lambda_{l} s\right)^{m_{l}}} .
$$

In the case where $p_{i} \sigma_{i}^{2}$ 's are all distinct, a more explicit expression for $f\left(\gamma_{e}\right)$ can be obtained. Thus, using [10, (4.331.2)], we obtain the closed-form expression of the ergodic mutual information over independent and distinctly distributed (i.d.d) channels as

$$
\bar{I}(\mathbf{p})=\frac{1}{\ln 2} \sum_{i=1}^{N_{t}} B_{i} \exp \left(\frac{N_{0}}{p_{i} \sigma_{i}^{2}}\right) E_{1}\left(\frac{N_{0}}{p_{i} \sigma_{i}^{2}}\right),
$$

where $E_{1}(\cdot)$ is the exponential integral function defined as $E_{1}(x)=\int_{x}^{\infty} e^{-t} / t d t$, for $x>0$, and $B_{i}$ is given by

$$
B_{i}=\prod_{m \neq i} \frac{p_{i} \sigma_{i}^{2}}{p_{i} \sigma_{i}^{2}-p_{m} \sigma_{m}^{2}}
$$

We now study the power allocation $\mathbf{p}$ that can maximize the ergodic mutual information $\bar{I}(\mathbf{p})$. The expression of $B_{i}$ in (7) makes it difficult to maximize $\bar{I}(\mathbf{p})$ with respect to $\mathbf{p}$. Here, we consider the simplest case where only two transmit antennas $\left(N_{t}=2\right)$ are used. After that, we propose a suboptimal power allocation scheme for multiple antennas based on the results obtained for two-antenna systems.

\section{B. Power allocation for two-transmit-antenna system}

For a system with only two transmit antennas, the problem of maximizing $\bar{I}(\mathbf{p})$ is equivalent to

$\max _{0 \leq p_{1} \leq 1}\left\{B_{1} e^{\frac{N_{0}}{p_{1} \sigma_{1}^{2}}} E_{1}\left(\frac{N_{0}}{p_{1} \sigma_{1}^{2}}\right)+B_{2} e^{\frac{N_{0}}{\left(1-p_{1}\right) \sigma_{2}^{2}}} E_{1}\left(\frac{N_{0}}{\left(1-p_{1}\right) \sigma_{2}^{2}}\right)\right\}$

It is shown in Appendix A that the optimization problem (8) is convex. We can solve it by letting its first derivative with respect to $p_{1}$ be zero. Note that when no such a $p_{1} \in[0,1]$ that can make the first derivative zero exists, the objective function in (8) degenerates to a monotonic function of $p_{1}$. In other words, one of the two ends of $p_{1}$ 's range should be the optimum value. Consequently, one antenna should be turned off. Hence, two different cases regarding power allocation are analyzed.

In the first case, both antennas are active. Assume that the power on the first antenna is $0<p_{1}<1$ while on the second antenna it is $p_{2}=1-p_{1}$. Taking the first derivative of objective function in (8) with respect to $p_{1}$ and equating it to zero, after applying high signal-to-noise ratio (SNR) assumption, we obtain

$$
\ln \frac{\left(1-p_{1}\right) \sigma_{2}^{2}}{p_{1} \sigma_{1}^{2}}=\frac{\left(\sigma_{1}^{2}+\sigma_{2}^{2}\right)\left(\sigma_{2}^{2}-p_{1}\left(\sigma_{2}^{2}+\sigma_{1}^{2}\right)\right)}{\sigma_{1}^{2} \sigma_{2}^{2}} .
$$

The details are also shown in Appendix A. Though $p_{1}$ as the solution to (9) cannot be obtained in closed form, we still can point it out by finding the cross point of the two curves which are the left and right sides of (9), respectively. We prove in Appendix B that there always exists one tangent point and at most one cross point for the two curves. The tangent point is $p_{1}=\sigma_{2}^{2} /\left(\sigma_{1}^{2}+\sigma_{2}^{2}\right)$, but it is not valid to be the optimal solution, since it violates the assumption of $p_{1} \sigma_{1}^{2} \neq p_{2} \sigma_{2}^{2}$ made in (6). After plotting the two curves, we can find that $p_{1}>1 / 2$ when $\sigma_{1}^{2}>\sigma_{2}^{2}$ and $p_{1}$ increases when the ratio $\xi_{12}=\sigma_{1}^{2} / \sigma_{2}^{2}$ increases. Moreover, there does not exist any cross point any more when the difference between channel conditions is large enough. In other words, valid solution $0<p_{1}<1$ to (9) does not exist for highly unbalanced channels. This leads to the second case where only one antenna is active.

When only one antenna is active, the problem of maximizing $\bar{I}(\mathbf{p})$ is trivial. It is clear that the total power should be assigned to the antenna with larger channel variance.

Based on the discussion above, the asymptotic power allocation scheme at high SNR only depends on the channel ratio $\xi_{12}=\sigma_{1}^{2} / \sigma_{2}^{2} \geq 1$. In Fig. 1, we plot the optimal solution (obtained by graphic method) of $p_{1}$ as a function of $\xi_{12}$ based on (9). It is seen that $p_{1}$ can be well approximated by

$$
p_{1}=f\left(\xi_{12}\right)=\left\{\begin{array}{l}
1-\frac{1}{2} \exp \left(-\xi_{12}+1\right), \quad 1 \leq \xi_{12} \leq \xi_{T} \\
1, \quad \xi_{12}>\xi_{T}
\end{array}\right.
$$

where the ratio threshold $\xi_{T}$ can be chosen larger than 10 .

\section{Power allocation for multiple-transmit-antenna system}

For multiple-antenna systems, it is difficult to directly optimize (6) with respect to p. Motivated by the results obtained for two-antenna systems, that is, more power on better channel, we propose a sub-optimal, but simple power allocation scheme which can provide near-optimal performance. The power on each antenna is assigned sequentially. In other words, $p_{1}$ is assigned first, afterward $p_{2}$ is assigned till $p_{N_{t}}$. When computing $p_{i}$, we spilt the $N_{t}-i+1$ antennas, which have not been assigned powers, into two groups. The channel gain with the first group is $\sigma_{i}^{2}$ and with the second group they are $\left\{\sigma_{i+1}^{2}, \cdots, \sigma_{N_{t}}^{2}\right\}$. Define $\sigma_{i+1}^{2(\mathrm{e})}$ as the equivalent channel 


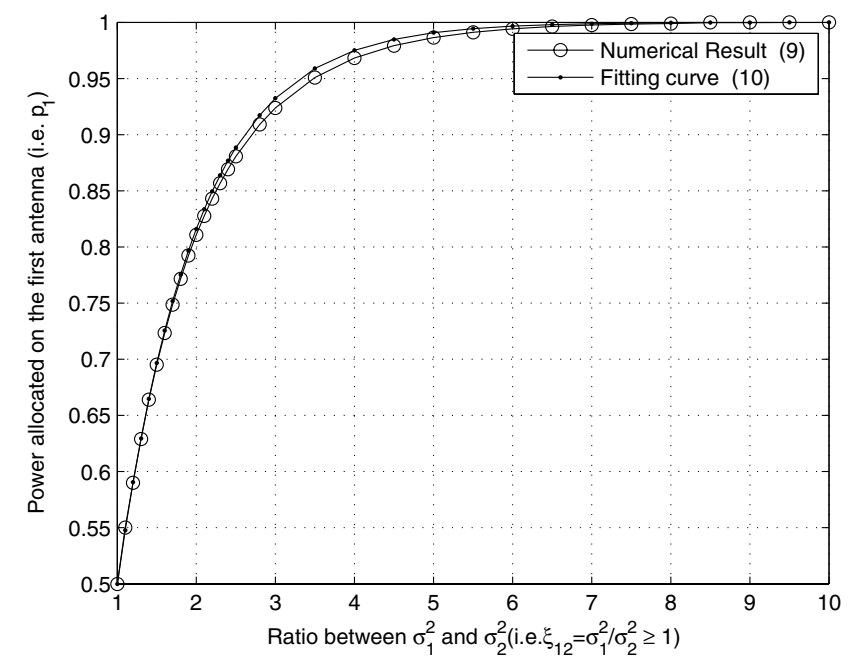

Fig. 1. Curve fitting

gain of the second group, whose exact definition is to be determined. A proposed power allocation scheme is

$$
p_{i}=\left\{\begin{array}{l}
\left(1-\sum_{k=1}^{i-1} p_{k}\right) f\left(\sigma_{i}^{2} / \sigma_{i+1}^{2(\mathrm{e})}\right), \quad \sigma_{i}^{2} \geq \sigma_{i+1}^{2(\mathrm{e})} \\
\left(1-\sum_{k=1}^{i-1} p_{k}\right)\left[1-f\left(\sigma_{i+1}^{2(\mathrm{e})} / \sigma_{i}^{2}\right)\right], \sigma_{i+1}^{2(\mathrm{e})}>\sigma_{i}^{2}
\end{array},\right.
$$

for $i=1, \cdots, N_{t}$.

We now propose a novel definition for $\sigma_{i+1}^{2(\text { e) }}$. First we compare each channel gain of $\left\{\sigma_{i+1}^{2}, \cdots, \sigma_{N_{t}}^{2}\right\}$ with $\sigma_{i}^{2}$ to form the set $\mathbf{S}_{i+1}$ in which each channel gain is larger than $\sigma_{i}^{2} / \xi_{T}$, i.e.,

$$
\left\{\sigma_{k}^{2} \in \mathbf{S}_{i+1} \mid \sigma_{i}^{2} / \sigma_{k}^{2} \leq \xi_{T}, k \in\left[i+1, N_{t}\right]\right\} .
$$

Assume there are $K_{i+1}$ elements in $\mathbf{S}_{i+1}$. We simply regard those $K_{i+1}$ associated antennas as one single antenna and define its equivalent channel gain $\sigma_{i+1}^{2(e)}$ as the norm of the vector $\left[\sigma_{i+1}^{2}, \sigma_{i+2}^{2}, \cdots, \sigma_{i+K_{i+1}}^{2}\right]$, i.e.

$$
\sigma_{i+1}^{2(\mathrm{e})}=\sqrt{\sum_{k=i+1}^{i+K_{i+1}}\left(\sigma_{k}^{2}\right)^{2}}
$$

Though we cannot prove it rigorously, the proposed equivalent channel gain in (12) together with (11) provides a near capacity-achieving performance for both slightly unbalanced and highly unbalanced channels which will be shown in Section V.

\section{OUTAGE PROBABILITY AND POWER ALLOCATION}

Given the instantaneous mutual information $I(\mathbf{p}, \mathbf{h})$ defined in (1) and an outage mutual information $I_{\text {out }}$, the outage probability is defined as

$$
P_{\text {out }}(\mathbf{p})=P\left(I(\mathbf{p}, \mathbf{h})<I_{\text {out }}\right)=P\left(\gamma_{e}<\gamma_{\text {out }}\right),
$$

where $\gamma_{\text {out }}=2^{I_{\text {out }}}-1$. Hence, the outage probability is the same as the cumulative distribution function (CDF) of $\gamma_{e}$, which is expressed as [9, (32)]

$$
P_{\text {out }}(\mathbf{p})=1+\left.\sum_{k} \frac{e^{-x / \lambda_{k}} \hat{g}_{k}^{\left(m_{k}-1\right)}(0, x)}{\left(-\lambda_{k}\right)^{m_{k}}\left(m_{k}-1\right) !}\right|_{x=\gamma_{\text {out }}} .
$$

Here $\hat{g}_{k}(s, x)$ is given by

$$
\hat{g}_{k}(s, x)=-\lambda_{k} e^{-s x} \prod_{l} \frac{1}{\left(\alpha_{k l}-\lambda_{l} s\right)^{m_{k l}}},
$$

with $\alpha_{k k} \triangleq-1, m_{k l}=m_{l}$ for $l \neq k$, and $m_{k k}=1$. In the case where $p_{i} \sigma_{i}^{2}$ 's are all distinct (i.e., i.d.d channels), the outage probability in (14) can be simplified to

$$
P_{\text {out }}(\mathbf{p})=\sum_{i=1}^{N_{t}} B_{i}\left(1-\exp \left(-\frac{N_{0} \gamma_{\text {out }}}{p_{i} \sigma_{i}^{2}}\right)\right),
$$

which is consistent with [7, (2)]. In [11], the authors derived the outage probability for DF cooperative communications. When assuming the source-relay link to be error free, we can verify that $[11,(10 \mathrm{a})]$ reduces to $(15)$.

In the following we derive a suboptimal power allocation scheme that can minimize an upper bound of the outage probability at any given $I_{\text {out }}$. By applying the Chernoff bound, the outage probability in (13) can be upper-bounded by

$$
P_{\text {out }}(\mathbf{p}) \leq E\left[e^{u\left(\gamma_{\text {out }}-\gamma_{e}\right)}\right]=e^{u \gamma_{\text {out }}} \prod_{i=1}^{N_{t}} \frac{1}{1+u p_{i} \sigma_{i}^{2} / N_{0}},
$$

where $u$ is a non-negative constant and can be chosen to optimize the tightness of the bound. Nevertheless, we choose $u=N_{t} / \gamma_{\text {out }}$ for simplicity. This bound can be minimized with respect to $p_{i}$ by maximizing $\prod_{i=1}^{N_{t}}\left(1+u p_{i} \sigma_{i}^{2} / N_{0}\right)$. Using the Lagrange method, we obtain the water-filling based suboptimal power allocation:

$$
p_{i}=\left\{1 / v-N_{0} \gamma_{\text {out }} /\left(\sigma_{i}^{2} N_{t}\right)\right\}^{+},
$$

where $\{a\}^{+}$denotes $\max \{0, a\}$. According to the properties of water filling, at high transmit SNR, the power tends to be equally allocated among all antennas, while at low SNR some of the antennas whose channel variances are significantly lower than the others may be turned off. These conclusions are consistent with the heuristic power control scheme in [7].

\section{NumericAl RESUltS}

We consider a system with 3 transmit antennas and 1 receive antenna. Both highly and slightly unbalanced channel conditions are discussed. Condition 1 is with parameter $\sigma_{1}^{2}=$ $300 / 111, \sigma_{2}^{2}=30 / 111$ and $\sigma_{3}^{2}=3 / 111$. Condition 2 is with parameter $\sigma_{1}^{2}=18 / 11, \sigma_{2}^{2}=9 / 11$ and $\sigma_{3}^{2}=6 / 11$. The sum of channel gains in both cases is equal to $N_{t}=3$.

In Fig. 2, the ergodic mutual information using different power allocations is illustrated. The result of the optimal power allocation is obtained using two-dimensional exhaustive search. It is seen that the proposed suboptimal scheme (11) performs almost the same as the optimal one and hence is near capacity-achieving. Also, 3-dB and 1-dB SNR gains are achieved over equal power allocation in the two channel 


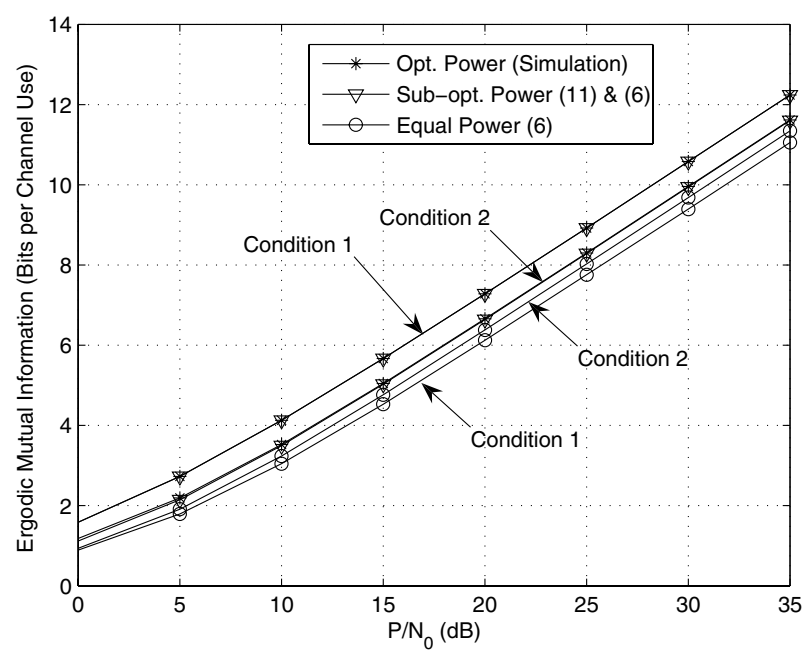

Fig. 2. Ergodic mutual information with different power allocations

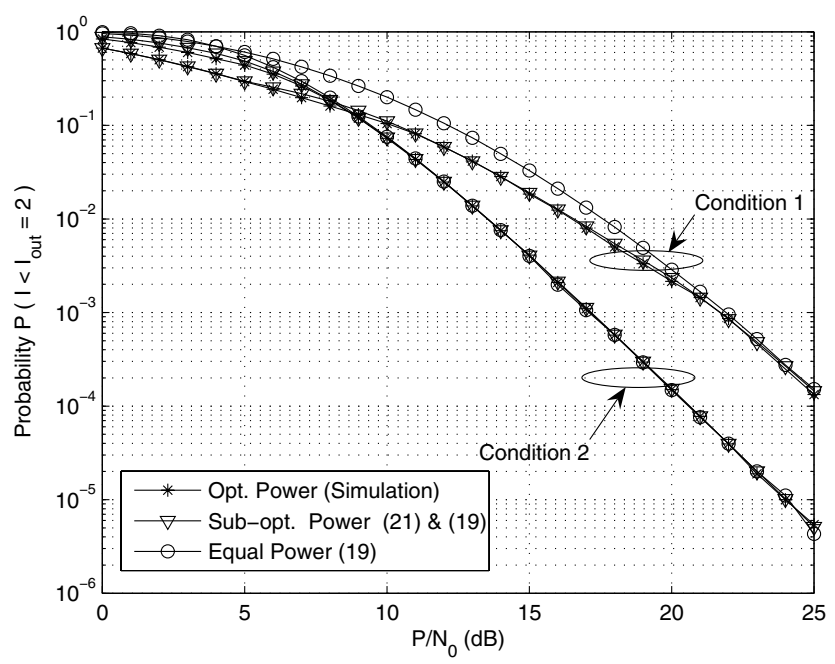

Fig. 3. Outage probability with different power allocations.

conditions, respectively. In addition, under the same total transmit SNR over channel condition 1, the mutual information obtained by the proposed power allocation is 1-bit/channel use higher compared with that of equal power allocation.

The outage probability for a given $I_{\text {out }}=2 \mathrm{bits} / \mathrm{channel}$ use is shown in Fig. 3. We can see that the proposed power allocation (17) provides performance very close to the optimal scheme (via exhaustive search).

Finally we compare in Figs. 4 and 5 the power values assigned to each antenna using the different criteria (11) and (17). The results show that, for ergodic mutual information maximization, the power allocation is independent of the total transmit SNR and only depends on the channel variances. Only one antenna is active if one channel variance is significantly larger than the others. And for slightly unbalanced channels all the antennas are active and more power is assigned to antennas with larger channel variance. For outage minimization, it follows the water-filling principle. When the total transmit SNR is high enough, all the antennas need to be active and

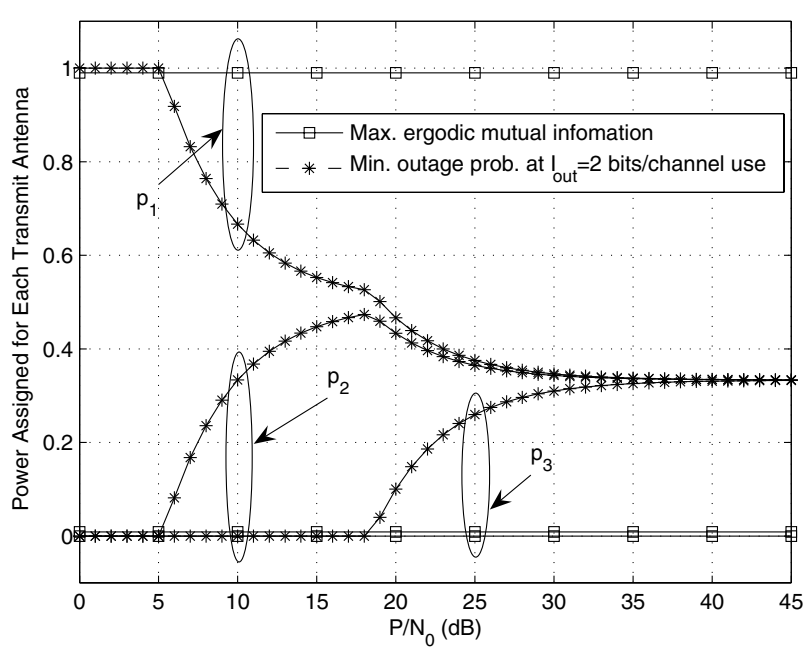

Fig. 4. Power values using different criteria under condition 1.

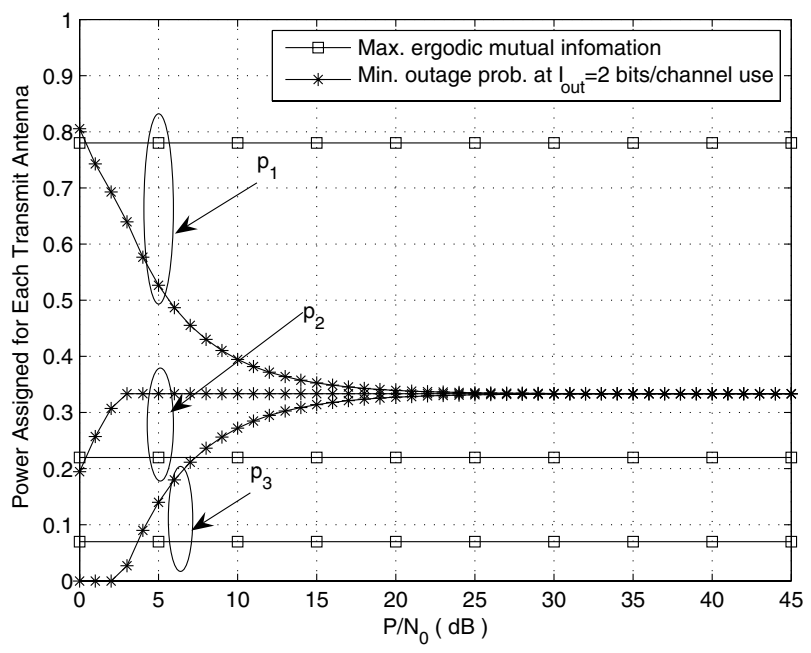

Fig. 5. Power values using different criteria under condition 2.

the power tends to be equally allocated.

\section{CONCLUSION}

We studied the capacity over non-identical MISO Rayleigh fading channels. Closed-form expressions of ergodic mutual information and outage probability are obtained. In addition, we derived suboptimal power allocation schemes which exploit the non-identical channel statistics. The power allocation scheme for maximizing the ergodic mutual information assigns more power to antennas with larger channel variances and outperforms equal power allocation considerably. For minimizing outage probability, the proposed power allocation follows the water-filling principle and brings performance gain over equal power allocation when the channels are highly unbalanced.

\section{APPENDIX A}

We first derive the first derivative of objective function in (8) with respect to $p_{1}$. After that we show that its second derivative with respect to $p_{1}$ is non-positive for $0 \leq p_{1} \leq 1$. Based 
on the identity $E_{1}(x)=-E_{i}(-x)=-\int_{-\infty}^{-x} \frac{e^{t}}{t} d t, \quad x>0$, let $A=B_{1} \exp \left(N_{0} /\left(p_{1} \sigma_{1}^{2}\right)\right), B=-E_{i}\left(-N_{0} /\left(p_{1} \sigma_{1}^{2}\right)\right), C=$ $B_{2} \exp \left(N_{0} /\left(\left(1-p_{1}\right) \sigma_{2}^{2}\right)\right)$ and $D=-E_{i}\left(-N_{0} /\left(\left(1-p_{1}\right) \sigma_{2}^{2}\right)\right)$. Taking the first derivative of $A, B, C$ and $D$ with respect to $p_{1}$, respectively, we get

$$
\begin{aligned}
A^{\prime} & =\frac{-\sigma_{1}^{2} \sigma_{2}^{2} e^{N_{0} /\left(p_{1} \sigma_{1}^{2}\right)} p_{1}-N_{0} e^{N_{0} /\left(p_{1} \sigma_{1}^{2}\right)}\left(p_{1} \sigma_{1}^{2}-\left(1-p_{1}\right) \sigma_{2}^{2}\right)}{p_{1}\left(p_{1} \sigma_{1}^{2}-\left(1-p_{1}\right) \sigma_{2}^{2}\right)^{2}} \\
B^{\prime} & =e^{-N_{0} /\left(p_{1} \sigma_{1}^{2}\right)} / p_{1} \\
C^{\prime} & =\frac{e^{N_{0} /\left(\left(1-p_{1}\right) \sigma_{2}^{2}\right)}\left(\left(1-p_{1}\right) \sigma_{1}^{2} \sigma_{2}^{2}+N_{0}\left(\left(1-p_{1}\right) \sigma_{2}^{2}-p_{1} \sigma_{1}^{2}\right)\right)}{\left(\left(1-p_{1}\right) \sigma_{2}^{2}-p_{1} \sigma_{1}^{2}\right)^{2}\left(1-p_{1}\right)} \\
D^{\prime} & =-e^{-N_{0} /\left(\left(1-p_{1}\right) \sigma_{2}^{2}\right)} /\left(1-p_{1}\right) .
\end{aligned}
$$

Note that $\lim _{x \rightarrow 0}-x E_{i}(-x)=0$ and $\lim _{N_{0} \rightarrow 0} E_{1}\left(N_{0} /\left(1-p_{1}\right) \sigma_{2}^{2}\right)-$ $E_{1}\left(N_{0} / p_{1} \sigma_{1}^{2}\right)=\ln \frac{\left(1-p_{1}\right) \sigma_{2}^{2}}{p_{1} \sigma_{1}^{2}}$. When assuming the SNR is very large (i.e. $N_{0} \rightarrow 0$ ), the first derivative of objective function in (8) (i.e. $A^{\prime} B+A B^{\prime}+C^{\prime} D+C D^{\prime}$ ) comes to

$$
\frac{\sigma_{1}^{2} \sigma_{2}^{2}\left(\ln \frac{\left(1-p_{1}\right) \sigma_{2}^{2}}{p_{1} \sigma_{1}^{2}}\right)}{\left(\left(1-p_{1}\right) \sigma_{2}^{2}-p_{1} \sigma_{1}^{2}\right)^{2}}-\frac{\sigma_{1}^{2}+\sigma_{2}^{2}}{\left(1-p_{1}\right) \sigma_{2}^{2}-p_{1} \sigma_{1}^{2}} .
$$

By letting (19) be zero, after simple manipulation, equation (9) can be obtain.

Having the first derivative, we can obtain the second derivative as follows. Differentiating (19) with respect to $p_{1}$, and multiplying the result by a positive term $\left\{\left[\left(1-p_{1}\right) \sigma_{2}^{2}-\right.\right.$ $\left.\left.p_{1} \sigma_{1}^{2}\right]^{2} p_{1}\left(1-p_{1}\right)\right\}^{-1}$, we obtain

$$
\begin{aligned}
y= & -\sigma_{1}^{2} \sigma_{2}^{2}+\frac{2 \sigma_{1}^{2} \sigma_{2}^{2}\left(\sigma_{1}^{2}+\sigma_{2}^{2}\right) p_{1}\left(1-p_{1}\right)}{\left(1-p_{1}\right) \sigma_{2}^{2}-p_{1} \sigma_{1}^{2}} \ln \frac{\left(1-p_{1}\right) \sigma_{2}^{2}}{p_{1} \sigma_{1}^{2}} \\
& -p_{1}\left(1-p_{1}\right)\left(\sigma_{1}^{2}+\sigma_{2}^{2}\right)^{2}
\end{aligned}
$$

Without loss of generality, by assuming $\xi=\sigma_{1}^{2} / \sigma_{2}^{2} \geq 1$ and the sum of channel gains to be $N_{t}$ (in this case $N_{t}=2$ ), one has $\sigma_{1}^{2}=2 \xi /(\xi+1)$ and $\sigma_{2}^{2}=2 /(\xi+1)$. Hence, (20) can be expressed by a function of $\xi$ and $p_{1}$ shown as

$$
y=\frac{-4 \xi}{(1+\xi)^{2}}+\frac{4 \times 2 \xi p_{1}\left(1-p_{1}\right)}{(\xi+1)\left(1-p_{1}-\xi p_{1}\right)} \ln \frac{1-p_{1}}{\xi p_{1}}-4 p_{1}\left(1-p_{1}\right) .
$$

Now we need to show that $y$ is non-positive for $0 \leq p_{1} \leq 1$. In order to show $y \leq 0$, we consider three different cases. First, in the case of $x=\left(1-p_{1}\right) /\left(\xi p_{1}\right)=1$, term $\ln \frac{1-p_{1}}{\xi p_{1}} /(1-$ $\left.p_{1}-\xi p_{1}\right)$ as part of the second term in (21) can be rewritten as $\ln x /\left(\xi p_{1} x-\xi p_{1}\right)$. By applying L'Hospital principle it is easy to show $y=0$. In the second case of $x=\left(1-p_{1}\right) /\left(\xi p_{1}\right)>1$, we substitute $p_{1}=1 /(x \xi+1)$ into (21) and get

$$
y=\frac{-4 \xi}{(\xi+1)^{2}}+\frac{4 \times 2 \xi x \ln (x)}{(\xi+1)(x-1)(\xi x+1)}-\frac{4 \xi x}{(\xi x+1)^{2}} .
$$

Since $x>1$, multiplying the two sides of (22) by positive term $(1+\xi)(\xi x+1)(x-1) /(4 \xi x)$, we get

$$
y_{1}=\frac{-(\xi x+1)(x-1)}{(\xi+1) x}-\frac{(x-1)(\xi+1)}{\xi x+1}+2 \ln x .
$$

By differentiating (23) with respect to $x$ and multiplying the result by positive term $x^{2}(1+\xi)(\xi x+1)^{2}, y_{1}$ comes to

$$
y_{2}=-(x-1)^{2}\left(x^{2} \xi^{3}+1\right) \text {. }
$$

Considering the property of quartic function $(\xi \geq 1)$ and only one real $\operatorname{root} x=1$ of (24), we can find that (23) is a monotonic decreasing function of $x$. Hence, $y_{1}=0$ is global maximum at point $x=1$. For the third case $0 \leq x<1$, the same method used here is applicable to show $y \leq 0$.

\section{APPENDIX B}

We will show that there always is one tangent point and at most one cross point for the two curves in (9). First we denote $f_{1}\left(p_{1}\right)=\ln \left(\left(1-p_{1}\right) \sigma_{2}^{2} / p_{1} \sigma_{1}^{2}\right)$ and $f_{2}\left(p_{1}\right)=\left(\sigma_{1}^{2}+\right.$ $\left.\sigma_{2}^{2}\right)\left(\sigma_{2}^{2}-p_{1}\left(\sigma_{2}^{2}+\sigma_{1}^{2}\right)\right) / \sigma_{1}^{2} \sigma_{2}^{2}$, respectively. We can show that when $p_{1}>1 / 2$ function $f_{1}\left(p_{1}\right)$ is convex while $p_{1}<1 / 2$ function $f_{1}\left(p_{1}\right)$ is concave. Since $f_{2}\left(p_{1}\right)$ is a linear function, there are at most three values of $p_{1}$ which can make $f_{1}\left(p_{1}\right)=$ $f_{2}\left(p_{1}\right)$. In our case, two of the three values are always equal. In other words, there is one tangent point between $f_{1}\left(p_{1}\right)$ and $f_{2}\left(p_{1}\right)$, and it lies at $p_{1}=\sigma_{2}^{2} /\left(\sigma_{1}^{2}+\sigma_{2}^{2}\right)$. This point is the tangent point between the two curves since

$$
f_{i}^{\prime}\left(p_{1}=\frac{\sigma_{2}^{2}}{\sigma_{1}^{2}+\sigma_{2}^{2}}\right)=-\frac{\left(\sigma_{1}^{2}+\sigma_{2}^{2}\right)^{2}}{\sigma_{1}^{2} \sigma_{2}^{2}} \quad i=1,2 .
$$

Also, since $f_{1}\left(p_{1}\right)=f_{2}\left(p_{1}\right)$ holds when $p_{1}=\sigma_{2}^{2} /\left(\sigma_{1}^{2}+\sigma_{2}^{2}\right)$. However, the tangent point $p_{1}=\sigma_{2}^{2} / \sigma_{1}^{2}+\sigma_{2}^{2}$ is not the valid solution since $p_{1} \sigma_{1}^{2}=p_{2} \sigma_{2}^{2}$ when $p_{1}=\sigma_{2}^{2} / \sigma_{1}^{2}+\sigma_{2}^{2}$, which conflicts with the assumption made when obtaining (6).

\section{REFERENCES}

[1] J. N. Laneman and G. W. Wornell, "Distribued space-time-coded protocols for exploiting cooperative diversity in wireless networks," IEEE Trans. on Information Theory, vol. 49, 2003.

[2] M. V. Clark, T. M. W. III, L. J. Greenstein, J. R. A, V. Erceg, and R. S. Roman, "Distributed versus centralized antenna arrays in broadband wireless networks," in Proc. IEEE VTC, vol. 1, 2001.

[3] J. He and P. Y. Kam, "On the performance of orthogonal spacetime block codes over independent, nonidentical Rayleigh/Ricean fading channels," in Proc. IEEE Globalcom, Nov 2006, pp. 1-5.

[4] H. Zhao, Y. Gong, Y. L. Guan, C. L. Law, and Y. Tang, "Spacetime block codes in Nakagami fading channels with non-identical mdistributions," in Proc. WCNC, Mar 2007, pp. 536-540.

[5] M. Tao and P. Y. Kam, "Analysis of differential orthogonal space-time block codes over semi-identical MIMO fading channels," IEEE Trans. Commun., pp. 282-291, Feb 2007.

[6] E. Abbe, E. Telatar, and L. Zheng, "The algebra of MIMO channels," in Allerton Annual Conference on Communication, Control and Computing, Oct 2005.

[7] J. Luo, R. S. Blum, L. Cimini, L. Greenstein, and A. Haimovich, "Power allocation in a transmit diversity system with mean channel gain information," IEEE Communications Letters, vol. 9, no. 7, 2005.

[8] E. Telatar, "Capacity of multi-antenna Gaussian channles," European Transaction on Telecommunication, vol. 10, no. 6, 1999.

[9] D. Raphaeli, "Distribution of noncentral indefinite quadratic forms in complex normal variables," IEEE Trans. on Info. Theory, vol. 42, 1996.

[10] I. S. Gradshteyn and I. M. Ryzhik, Table of Integrals, Series, and Products. Academic Press Limited, 1994.

[11] N. C. Beaulieu and J. Hu, "A closed-form expression for the outage probability of decode-and-forward relaying in dissimilar rayleigh fading channels," IEEE Communications Letters, vol. 10, 2006. 